\title{
THE EQUIVALENCE THEOREM FOR CHIRAL LAGRANGIANS
}

\author{
Antonio DOBADO 卢 and José Ramón PELAEZ \\ Departamento de Física Teórica \\ Universidad Complutense de Madrid \\ 28040 Madrid, Spain \\ $\mathrm{FT} / \mathrm{UCM} / 2 / 94$ \\ hep-ph 9404239
}

April 1994

\begin{abstract}
In this work we derive the version of the Equivalence Theorem that applies when the symmetry breaking sector of the Standard Model is described by a general chiral lagrangian. The demonstration is valid for renormalized fields for any value of the gauge parameter (in $R_{\xi}$ gauges) and any parametrization of the coset space. It is based in the absence of gauge anomalies which makes it possible to build an (anti)BRS invariant chiral lagrangian in terms of the renormalized fields and therefore to use the corresponding Ward identities to obtain the theorem.
\end{abstract}

\footnotetext{
${ }^{1}$ E-mail: dobado@cernvm.cern.ch

${ }^{2}$ E-mail:pelaez@fis.ucm.es
} 


\section{Introduction}

This paper deals with the problem of finding a general derivation of the socalled Equivalence Theorem (ET) $[1,2,3]$ relating the $S$ matrix elements of electroweak gauge bosons longitudinal components with the corresponding $S$ matrix elements for the would be Goldstone Bosons (GB) at high energies compared with the electroweak symmetry breaking scale $v=250 \mathrm{GeV}$. This kind of relation could be very useful in order to obtain information about the Symmetry Breaking Sector (SBS) of the Standard Model (SM) from the future LHC data since computations are by far easier to be done for scalars than for longitudinal gauge bosons. Despite the very precise data collected at LEP we have virtually no information about the SBS of the SM and it is still a mystery whether this sector can be described by the Minimal Standard Model (MSM) with just one doublet of Higgs fields, the Minimal Supersymmetric SM (see [4] for a review), Technicolor [5] or other models like the so-called BESS model [6].

As it is not possible, at present, to know which is the dynamics responsible for the spontaneous breaking of the electroweak group $S U(2)_{L} \times U(1)_{Y}$ to the electromagnetic group $U(1)_{e m}$, it is very important to develop some model independent framework to describe, at least phenomenologically, the SBS mechanism. Recently such a framework has been proposed using a formalism borrowed from low-energy hadron physics and called Chiral Perturbation Theory $(\chi \mathrm{PT})[7]$. This approach has proved to be quite useful not only for the model independent description of longitudinal electroweak gauge bosons scattering (assuming the validity of the ET) [8] but also for the analysis of the precision measurements obtained at LEP [9].

To apply $\chi \mathrm{PT}$ to the description of the SBS of the SM one assumes that there must be some physical system coupled to the SM with a global symmetry breaking from a group $G$ to another group $H$ which drives the spontaneous symmetry breaking of the gauge group $S U(2)_{L} \times U(1)_{Y}$ to $U(1)_{\mathrm{em}}$ thus giving masses to the the $W^{ \pm}$and $Z$ gauge bosons through the standard Higgs mechanism. The GB related to the global $G$ to $H$ symmetry breaking live in the coset space $G / H$ and their low energy dynamics is described by a gauged Non-Linear Sigma Model supplemented with and infinite number of higher derivative terms (but finite for practical applications) needed for the renormalization of the model.

As we said above $\chi \mathrm{PT}$ has been used together with the ET to describe the scattering of the longitudinal components of the gauge bosons. However, no rigorous proof of this theorem has been presented until the present moment 
outside the framework of the MSM. Moreover, it has been realized recently that even in that case the simple original formulation of the SM should be corrected in order to take into account the different renormalization of the GB and the gauge bosons [10].

In this work we derive the general form of the theorem valid for a chiral lagrangian description of the SBS of the SM including those renormalization factors mentioned before. In so doing we complete the work presented in [11] where we derived the theorem for regularized Green functions but not for renormalized Green functions. Our derivation is based on the nice formal proof of the ET for the MSM by Chanowitz and Gaillard [2] which relies in the BRS symmetry [12] of the Green functions, but we take into account the peculiarities of $\chi \mathrm{PT}$ and include all the renormalization factors. To implement the BRS symmetry we follow a very general methods given in [13] that provides a very useful way to build, starting from a gauge invariant lagrangian, a quantum lagrangian which is (anti)-BRS invariant as well as a generalization of the standard Faddeev-Popov method, and therefore, valid for the definition of $\chi \mathrm{PT}$ when the gauge fields are quantized and included in loops. Note that this is not the case in the original applications of $\chi \mathrm{PT}$ to the description of the low-energy pion dynamics.

\section{The chiral lagrangian description of the SBS}

In order to choose the appropriate $G$ and $H$ groups we require the following conditions: a) $\operatorname{dim} K=\operatorname{dim} G / H=3$ as we need three GB to give mass to the three observed gauge bosons $W^{ \pm}$and $Z$; b) $G$ should contain the $S U(2)_{L} \times U(1)_{Y}$ group so that the symmetry breaking sector can couple to the electroweak gauge bosons; c) Since we want to ensure the experimental relation $\rho \simeq 1$, we will require the subgroup $H$ to contain the custodial group $S U(2)_{L+R}$ [14]. This automatically yields $\rho=1$ when the gauge fields are switched off and also implies that the photon will remain massless since $U(1)_{e m}$ is contained in $S U(2)_{L+R}$ and therefore in $H$. In [11] it was shown that these conditions completely determine the $G$ and $H$ groups to be $G=$ $S U(2)_{R} \times S U(2)_{L}$ and $H=S U(2)_{L+R}$ so that $K=G / H=S^{3}$.

Therefore, the most general dynamics of the symmetry breaking sector of the SM compatible with all known constrains can be defined as a gauged non-linear sigma model based on the coset space $K=G / H=$

$S U(2)_{L} \times S U(2)_{R} / S U(2)_{L+R}=S^{3}$ with gauge group $S U(2)_{L} \times U(1)_{Y}$. The 
corresponding lagrangian can be written as:

$$
\begin{aligned}
\mathcal{L}_{g} & =\mathcal{L}_{Y M}^{L}+\mathcal{L}_{Y M}^{Y} \\
& +\frac{1}{2} g_{\alpha \beta}(\omega) D_{\mu} \omega^{\alpha} D^{\mu} \omega^{\beta} \\
& + \text { higher covariant derivative terms }
\end{aligned}
$$

where $\mathcal{L}_{Y M}^{L}$ and $\mathcal{L}_{Y M}^{Y}$ are the Yang-Mills lagrangians for the $S U(2)_{L}$ and $U(1)_{Y}$ gauge fields $W_{\mu}^{a}$ and $B_{\mu}, \omega^{\alpha}$ are arbitrary coordinates chosen on the coset $S^{3}$ with coordinates $\omega^{\alpha}=0$ for the classical vacuum. The action of the group $G$ on this space defines the killing vectors $\xi_{a}^{\alpha}$ through $\delta \omega^{\alpha}=\xi_{a}^{\alpha}(\omega) \epsilon^{a}$ which is the non-linear transformation of the GB $\omega^{\alpha}(x)$ under the action of an infinitesimal $G$ element. Here, the $a$ index runs from 1 to 6 where the values 1 to 3 correspond to the unbroken $H=S U(2)_{L} \times S U(2)_{R}$ generators. The $S^{3}$ metrics $g_{\alpha \beta}$ is defined as follows: Let $e_{a}=e_{a}^{\alpha} \partial / \partial \omega^{\alpha}$ with $e_{a}^{\alpha}=\xi_{a+3}^{\alpha}$ for $a=1,2,3$ i.e. the dreibein is just the set of killing vectors corresponding to the 3 broken generators, then $g_{\alpha \beta}$ is the inverse of $g^{\alpha \beta}$ where $g^{\alpha \beta}=e_{a}^{\alpha} e^{\beta a}$. It is easy to show that $G$ is the isometry group of $S^{3}$ so that $g_{\alpha \beta}^{\prime}(\omega)=g_{\alpha \beta}(\omega)$ under any $G$ transformation. The covariant derivatives are defined as:

$$
D_{\mu} \omega^{\alpha}=\partial_{\mu} \omega^{\alpha}-g l_{a}^{\alpha} W_{\mu}^{a}-g^{\prime} y^{\alpha} B_{\mu}
$$

where $l_{a}^{\alpha}$ and $y^{\alpha}$ are the killing vectors corresponding to the gauged group $S U(2)_{L} \times U(1)_{Y}$. The higher derivative terms include in principle any covariant (in the space-time and the $S^{3}$ sense) piece with an arbitrary high number of covariant derivatives and arbitrary couplings so that we can reproduce any dynamics compatible with the symmetry breaking pattern $S U(2)_{L} \times$ $S U(2)_{R} / S U(2)_{L+R}=S^{3}$ and the gauge group $S U(2)_{L} \times U(1)_{Y}$. The gauge transformations are:

$$
\begin{aligned}
\delta \omega^{\alpha} & =l_{a}^{\alpha} \epsilon_{L}^{a}(x)+y^{\alpha} \epsilon_{Y}(x) \\
\delta W_{\mu}^{a} & =\frac{1}{g} \partial_{\mu} \epsilon_{L}^{a}(x)+\epsilon_{a b c} W_{\mu a} \epsilon_{L c}(x) \\
\delta B_{\mu} & =\frac{1}{g^{\prime}} \partial_{\mu} \epsilon_{Y}(x)
\end{aligned}
$$

\section{The BRS transformations and the quan- tum lagrangian}

The above gauge transformations satisfy the well known properties of closure (since the Lie brackets of the gauged Killing vectors satisfy the correspond- 
ing $S U(2)_{L} \times U(1)_{Y}$ algebra) and the Jacobi identity. Following [13] this fact makes it possible to build the related (anti)-BRS transformations by introducing the anti-commuting ghost fields $c_{a}$ and $\bar{c}_{a}$, and the commuting auxiliary field $B_{a}$ with $a=1,2,3,4$. In the following we will use an unified notation where the first three values of the gauge indices $a=1,2,3$ refer to the $S U(2)_{L}$ group and $a=4$ refers to $U(1)_{Y}$ so that the gauge field $W_{\mu}^{a}$ with $a=1,2,3,4$ will be defined as $W_{\mu}^{a}=W_{\mu}^{a}$ for $a=1,2,3$ and $W_{\mu}^{4}=B_{\mu}$. In addition we introduce the Killing vector $L_{a}^{\alpha}$ with $a=1,2,3,4$ as $L_{a}^{\alpha}=g l_{a}^{\alpha}$ for $a=1,2,3$ and $L_{4}^{\alpha}=g^{\prime} y^{\alpha}$ and the completely antisymmetric symbols $f_{a b c}$ with $a=1,2,3,4$ as $f_{a b c}=g \epsilon_{a b c}$ for $a=1,2,3$ and $f_{a b 4}=0$.

The closure relation and the Jacobi identity are equivalent to the nilpotency properties of the $(\bar{s})-s$ of the corresponding (anti)-BRS transformations obtained using the general method of [13]

$$
s^{2}=s \bar{s}+\bar{s} s=\bar{s}^{2}=0
$$

These nilpotency properties are very important to define a quantum lagrangian (anti)-BRS invariant as

$$
\mathcal{L}_{Q}=\mathcal{L}_{g}+\frac{1}{2} s \bar{s}\left[W_{\mu}^{a} W^{\mu a}+2 \xi f(\omega)+\xi c^{a} \bar{c}_{a}\right]
$$

where $f$ is any scalar analytical function with $\partial f(\omega) / \partial \omega^{\alpha}=\omega^{\alpha}+O\left(\omega^{2}\right)$. The new term added to the gauge invariant lagrangian (see [11] for complete display) can be understood as a generalization of the more usual gauge fixing and Faddeev-Popov terms corresponding to the t'Hooft like gauges $\left(R_{\xi}\right.$ gauges) which have two main advantages: First, they provide a contribution to the quadratic part in the gauge fields of the lagrangian so that the corresponding operator can be inverted giving rise to well defined $R_{\xi}$ propagators to be used in perturbation theory. Second, these gauges cancel the unwanted GB and gauge boson mixing terms appearing in the third term on eq. 1 through the covariant derivatives. In addition, this generalized method produces other GB-gauge boson and ghost-gauge boson interactions. For gauges different from that of Landau $(\xi=0)$ we also have quartic ghost interactions and GB-ghosts interactions.

From the (anti)-BRS invariance of the lagrangian in eq.5 it is possible to derive the corresponding Ward identities for the dimensionally regularized Green functions that are used in the proof of the ET. Note that the use of dimensional regularization is needed not only to preserve the (anti)-BRS invariance in the regularized lagrangian but also to avoid the $-\frac{i}{2} \delta^{n}(0) \operatorname{tr} \log g$ 
term that would otherwise appear in the quantum lagrangian of the nonlinear sigma model (NLSM) coming from the path integral measure of the GB fields [15] .

However, in practice one is not only interested in the regularized Green functions but also in the renormalized Green functions in order to make predictions for the different physical processes. To cancel all the divergences appearing in the Green functions obtained from the lagrangian in eq.5 one needs to consider the renormalized lagrangian which consists on that of eq. 5 plus other terms with the corresponding couplings needed to reproduce all the divergent structures appearing in the Green functions. The precise form of these counterterms is not known beyond those with four derivatives [16], but in any case, they should be (anti)-BRS invariant too. Otherwise, the gauge invariance of the model would be anomalous, i.e., broken by quantum effects. However, even when we have chiral fermions coupled to GB and gauge bosons, it is well known that the standard hypercharge assignments in the SM and the fact that the number of colors is $N_{c}=3$ are such that all possible gauge and mixed gauge-gravitational anomalies cancel, including the non-perturbative $S U(2)$ discovered by Witten [17]. In addition there are potential reparametrization invariance anomalies that could break the invariance under changes of coordinates on the coset, but as it was shown in [18] these anomalies are absent from NLSM defined on spaces of dimension lower than the space-time dimension as it happens in the case here considered.

Therefore, when we take into account all the terms needed, we obtain a lagrangian with an infinite number of terms which is (anti)-BRS invariant. It can be understood as the renormalized lagrangian of a renormalizable theory (but with an infinite number of couplings) written in terms of the bare fields an couplings. We can also give this renormalized lagrangian using the renormalized fields and couplings. The terms appearing in this case have the same form (as the theory is renormalizable in the generalized sense described above) but now some $Z$ factors appear in front of them. The relation between the renormalized and the bare fields and gauge couplings is given by:

$$
\begin{gathered}
W_{0 \mu}^{a}(x)=Z_{3}^{(a) 1 / 2} W_{\mu}^{a}(x) ; \pi_{o}^{\alpha}(x)=Z_{\pi}^{(\alpha) 1 / 2} \pi^{\alpha}(x) ; g_{0}^{(a)}=Z_{g}^{(a)} g^{(a)} ; \xi_{0}^{(a)}=Z_{3}^{(a)} \xi^{(a)} \\
c_{0}^{a}(x)=\widetilde{Z}_{2}^{(a) 1 / 2} c^{a}(x) ; \bar{c}_{0}^{a}(x)=\widetilde{Z}_{2}^{(a) 1 / 2} \bar{c}^{a}(x) ; B_{0}^{a}(x)=\widetilde{Z}_{2}^{(a)} B^{a}(x) ; v_{0}=Z_{v}^{1 / 2} v
\end{gathered}
$$

where $g^{(a)}=g$ for $a=1,2,3$ and $g^{(4)}=g^{\prime}$. The first three $Z_{3}$ are equal due to the gauge structure of the model. Note that from now on we use as a notation that the indices between parenthesis are not summed and that those 
fields and constants without 0 subscripts refer to the renormalized ones. In addition we also have infinite relations between the bare and the renormalized couplings appearing in the chiral lagrangian. Due to the existence of an (anti)-BRS symmetry in the renormalized lagrangian written in terms of the bare quantities it is possible to find the corresponding "renormalized" (anti)BRS transformations which will leave invariant the renormalized lagrangian once written in terms of the renormalized fields and couplings. Those (anti)BRS transformations can be written as:

$$
\begin{array}{ll}
s_{R}\left[\omega^{\alpha}\right]=X^{(a)} L_{R a}^{\alpha} c^{a} & \bar{s}_{R}\left[\omega^{\alpha}\right]=X^{(a)} L_{R a}^{\alpha} \bar{c}^{a} \\
s_{R}\left[W^{\mu a}\right]=X^{(a)} D_{R c}^{\mu a} c^{c} & \bar{s}_{R}\left[W^{\mu a}\right]=X^{(a)} D_{R c}^{\mu a} \bar{c}^{c} \\
s_{R}\left[c^{a}\right]=-\frac{X^{(a)}}{2} f_{R b c}^{a} c^{b} c^{c} & \bar{s}_{R}\left[c^{a}\right]=-\frac{X^{(a)}}{2} f_{R b c}^{a} \bar{c}^{b} c^{c} \\
s_{R}\left[\bar{c}^{a}\right]=X^{(a)} \frac{B^{(a)}}{\sqrt{\xi^{(a)}}} & \bar{s}_{R}\left[\bar{c}^{a}\right]=-X^{(a)}\left(\frac{B^{(a)}}{\sqrt{\xi^{(a)}}}+f_{R b c}^{a} \bar{c}^{b} c^{c}\right) \\
s_{R}\left[B^{a}\right]=0 & \bar{s}_{R}\left[B^{a}\right]=-X^{(a)} f_{R b c}^{a} \bar{c}^{b} B^{c}
\end{array}
$$

where $L_{R a}^{\alpha}=Z_{\pi}^{(\alpha)-1 / 2} Z_{3}^{(a) 1 / 2} L_{a}^{\alpha}$ and $f_{R b c}^{a}=Z_{g}^{(a)} Z_{3}^{(a) 1 / 2} g f_{b c}^{a}$.

Once we have a set of (anti)-BRS symmetry transformations for the renormalized lagrangian in terms of the renormalized fields we can apply standard methods to obtain the corresponding Ward identities for the renormalized Green functions. In particular, some of those relations will be used to find the version of the ET that applies to the chiral lagrangian description of the symmetry breaking sector of the SM.

\section{Ward Identities}

The ET provides the relationship between $S$-matrix elements involving an arbitrary number of longitudinal gauge bosons $W_{L}$ and those elements with all the external $W_{L}$ replaced by their corresponding GB. To find that relation, and following the steps of the Chanowitz-Gaillard proof [2], we will make use of the renormalized lagrangian BRS invariance to derive Ward identities relating the desired renormalized connected Green's functions, that will be converted in relations between $S$-matrix elements by means of the LehmannSymanzik-Zimmermann (LSZ) reduction formula.

Indeed, the renormalized effective action $\Gamma_{R}[A]$ obtained from the quantum lagrangian of the gauged NLSM is (anti-) BRS invariant too. ( $A$ stands 
for any field appearing in the quantum lagrangian i.e. $\left.A_{i}=\omega^{\alpha}, W_{\mu}^{a}, c^{a}, \bar{c}^{a}, B^{a}\right)$. This invariance can be stated as follows:

$$
\sum_{i} \int d^{4} x s_{R}\left[A_{i}\right] \frac{\delta \Gamma_{R}[A]}{\delta A_{i}}=0
$$

The generating functional for renormalized connected Green's functions $W_{R}\left(x_{1}, \ldots, x_{n}\right)$ is given, in momentum space, by the following definition:

$$
W_{R}[J]=(2 \pi)^{4} \sum_{n=1} \int \prod_{i=1}^{n} \frac{d^{4} p_{i}}{(2 \pi)^{4}} \delta^{4}\left(\sum_{i} p_{i}\right) J_{i_{1}}\left(-p_{1}\right) \ldots J_{i_{n}}\left(-p_{n}\right) W_{R i_{1}, \ldots, i_{n}}\left(p_{1}, \ldots p_{n}\right)
$$

Using the well known relations:

$$
W_{R}[J]+\Gamma_{R}[A]+\sum_{i} \int d^{4} x J_{i}(x) A_{i}(x)=0 ; A_{i}(x)=-\frac{\delta W_{R}[J]}{\delta J_{i}(x)} ; J_{i}(x)=-\frac{\delta \Gamma_{R}[A]}{\delta A_{i}(x)}
$$

we can write the fields as follows:

$$
A_{i}(k)=(2 \pi)^{4} \sum_{n=1} \int \prod_{j=1}^{n} \frac{d^{4} p_{j}}{(2 \pi)^{4}} J_{i_{j}}\left(-p_{j}\right) W_{R i, i_{1}, \ldots, i_{n}}\left(k, p_{1}, \ldots p_{n}\right) \delta^{4}\left(k+\sum_{j} p_{j}\right)
$$

It is important to notice that all the terms in this expansion contain at least one current $J$. Gathering these expressions, the condition of BRS invariance for the effective action is written:

$$
\sum_{i} \int \frac{d^{4} k}{(2 \pi)^{4}} s_{R}\left[A_{i}(k)\right] J_{i}(-k)=0
$$

It is now straightforward to obtain Ward identities just by expanding the fields contained in $s_{R}\left[A_{i}(k)\right]$ in terms of connected Green's functions as in eq.11, and then taking functional derivatives with respect to $J_{i}(p)$ at $J=0$. Since we are mainly interested in relations concerning $W_{L}$ and GB, we need to consider Green functions involving the auxiliary $B$ field, as it is nothing but the gauge fixing condition that intuitively identifies $W_{L}$ and the GB. To illustrate the general procedure we derive now a Ward identity for the two-point Green function with one $B$ field. The result thus obtained will be used later in the complete proof for the modified ET. So we write:

$$
\left.\frac{\delta}{\delta J_{\bar{c}_{b}}(-k)} \frac{\delta}{\delta J_{j}(p)} \sum_{i} \int \frac{d^{4} k}{(2 \pi)^{4}} s_{R}\left[A_{i}(k)\right] J_{i}(-k)\right|_{J=0}=0
$$


The only possible contributions come from those terms in the BRS transformations involving just one current. Thus, from eqs.7 and 11 we obtain:

$$
\frac{X^{(b)}}{\sqrt{\xi^{(b)}}} W_{B^{b} l}(p)=-X^{(a)} D_{R l}^{a}(p) W_{c^{a} \bar{c}^{b}}(p)=
$$

where:

$$
D_{R l}^{a}(p)=i p_{\mu} \delta_{l}^{W_{\mu a}}+L_{R a}^{(0) \alpha} \delta_{l}^{\omega^{\alpha}}
$$

Here we can see two important differences with the formal proof of [2]: First, the $X$ factors coming from renormalization. Second, the $L_{R}$ term which is due to the nonlinear realization of the symmetry. Nevertheless, this term only contributes in the zeroth order $L_{R}^{(0)}$, thus simplifying the complicated relation between gauge and Goldstone bosons that one would expect naively from the nonlinear gauge fixing condition. We now want to obtain the general expression, and so we start from:

$$
\left.\frac{\delta}{\delta J_{\bar{c}_{a_{1}}}(-k)} \prod_{j=2}^{s} \frac{\delta}{\delta J_{B_{a_{j}}}\left(-k_{j}\right)} \prod_{k=1}^{m} \frac{\delta}{\delta J_{A_{k}}\left(-p_{k}\right)} \sum_{i} \int \frac{d^{4} q}{(2 \pi)^{4}} s_{R}\left[A_{i}(q)\right] J_{i}(-q)\right|_{J=0}=0
$$

Where we will impose that the currents $J_{A_{k}}$ are only associated to physical $A_{k}$ fields. We can easily see from the BRS transformations that we do not get any contribution if $A_{i}=B$, neither when $A_{i}=\omega, c$ since there are no $J_{\omega}$ nor $J_{c}$ derivatives. Since the $A_{k}$ are physical, their polarization vectors will cancel the derivative term in $s_{R}\left[W_{\mu}^{a}\right]=i k_{\mu} c^{a}+\epsilon_{R b c}^{a} W_{\mu b} c_{c}$ because $\epsilon \cdot k_{\mu}=0$. Thus, we are only considering those terms coming from $s_{R}[\bar{c}]$ and the part which is left from $s_{R}\left[W_{\mu}^{a}\right]$ that we will call, generically, "bilinear terms". Finally we obtain:

$$
\frac{X^{\left(a_{1}\right)}}{\sqrt{\xi^{\left(a_{1}\right)}}} W_{B_{a_{1}} B_{a_{2}} \ldots B_{a_{s}} A_{1} \ldots A_{m}}\left(k_{1}, \ldots, k_{s}, p_{1}, \ldots p_{m}\right)+\text { bilinear terms }=0
$$

where $\sum_{i} k_{i}=-\sum_{i} p_{i}$. In order to translate this result to off-shell $S$-matrix elements, we apply the LSZ reduction formula:

$$
\begin{array}{r}
\frac{X^{\left(a_{1}\right)}}{\sqrt{\xi^{\left(a_{1}\right)}}}\left(\prod_{i=1}^{m} W_{A_{i} A_{i}}\left(p_{i}\right)\right) \sum_{l_{j}}\left(\prod_{j=1}^{s} W_{B_{a_{j}} l_{j}}\left(k_{j}\right)\right) \begin{array}{r}
S_{l_{1} . . l_{s} A_{1} \ldots A_{m}}^{\text {off-shell }} \\
+ \text { bilinear terms }
\end{array}=0 \\
\left.+k_{1} \ldots k_{s}, p_{1} \ldots p_{s}\right) \\
\end{array}
$$

As the $a_{1}$ index is free we can drop the factor $X / \sqrt{\xi}$ which is irrelevant. When we multiply the whole last equation by the inverse $A_{i}$ propagators, 
setting their momenta on shell, that is $p_{i}^{2}=m_{A_{i}}^{2}$, we can use the same argument as in [2] to cancel the "bilinear terms" since they are the product of two connected Green's functions contracted properly, but with one off-shell momentum, and without the pole needed to compensate for $W_{A_{i} A_{i}}^{-1}\left(p_{1}\right) \rightarrow 0$ when $p_{1}^{2}=m_{A_{1}}^{2}$. Therefore, using eq.14 to substitute the $B$ field two point functions, we obtain:

$$
\left.\sum_{l_{j}}\left(\prod_{j=1}^{s} \frac{\sqrt{\xi^{\left(a_{j}\right)}}}{X^{\left(a_{j}\right)}} X^{\left(c_{j}\right)} W_{c^{c_{j}} \bar{c}^{a_{j}}}\left(k_{j}\right) D_{R l_{j}}^{c_{j}}\left(k_{j}\right)\right) S_{l_{1} . . l_{s} A_{1} \ldots A_{m}}^{\text {off-shell }}\left(k_{1} \ldots k_{s}, p_{1} \ldots p_{s}\right)\right|_{p_{i}^{2}=m_{A_{i}}^{2}}=0
$$

Now, we can take away the $\sqrt{\xi^{\left(a_{j}\right)}} / X^{a_{j}}$ factors since the $a_{j}$ are not contracted. Then, we multiply by the ghost (non-diagonal, in principle) inverse two point functions $W_{c^{d} j \bar{c}^{a_{j}}}^{-1}\left(k_{j}\right)$ so that the $d_{j}$ index is again free allowing us to drop the last $X$ factor. Therefore, we arrive to the following expression:

$$
\left.\sum_{l_{1} \ldots l_{r}} \prod_{i=1}^{s} D_{R l_{i}}^{a_{i}}\left(p_{i}\right) S_{l_{1} . . l_{s}, A_{1} . . . A_{m}}^{o f f-s e l l}\left(p_{1} . . p_{r}, k_{1} . . k_{m}\right)\right|_{p_{i}^{2}=m_{A_{i}}^{2}}=0
$$

\section{The Equivalence Theorem}

Our aim is to obtain the $S$-matrix elements from this formulae by setting all the momenta on-shell, but the $W_{\mu}$ fields in the $D_{R}$ operator are not physical fields. We still have to obtain the physical combinations by means of a transformation $\widetilde{W}_{\mu}^{a}=R^{a b} W_{\mu}^{b}$, whose most general form will be :

$$
\left(\begin{array}{c}
\widetilde{W}_{\mu}^{1} \\
\widetilde{W}_{\mu}^{2} \\
\widetilde{W}_{\mu}^{3} \\
\widetilde{W}_{\mu}^{4}
\end{array}\right)=\left(\begin{array}{c}
W_{\mu}^{-} \\
W_{\mu}^{+} \\
Z_{\mu}^{\text {phys }} \\
A_{\mu}^{\text {phys }}
\end{array}\right)=\left(\begin{array}{cccc}
1 / \sqrt{2} & i / \sqrt{2} & 0 & 0 \\
1 / \sqrt{2} & -i / \sqrt{2} & 0 & 0 \\
0 & 0 & \cos \theta & -\sin \theta \\
0 & 0 & \sin \theta^{\prime} & \cos \theta^{\prime}
\end{array}\right)\left(\begin{array}{c}
W_{\mu}^{1} \\
W_{\mu}^{2} \\
W_{\mu}^{3} \\
W_{\mu}^{4}
\end{array}\right)
$$

These new fields are the renormalized fields which ensure that the poles of the exact propagators are located at the values of the corresponding physical masses . Once we have obtained them, we also define: $\widetilde{L}_{R \alpha}^{(0) a}=L_{R \alpha}^{(0) a}\left(R^{-1}\right)^{b a}$. Therefore we finally write:

$$
\sum_{l_{1} \ldots l_{r}} \prod_{i=1}^{s} \widetilde{D}_{R l_{i}}^{a_{i}}\left(p_{i}\right) S_{l_{1} . . l_{s}, A_{1} . . . A_{m}}\left(p_{1} . . p_{r}, k_{1} . . k_{m}\right)=0
$$


where

$$
\widetilde{D}_{R l}^{a}(p)=i p_{\mu} \delta_{l}^{\widetilde{W}_{R \mu a}}+\widetilde{L}_{R a}^{(0) \alpha} \delta_{l}^{\omega^{\alpha}}
$$

and we have set the $p_{i}$ momenta on-shell for the massive physical vector bosons.

The next step to obtain the modified version of the ET is to substitute the momenta in each $\widetilde{D}_{R l}^{a}(p)$ using the relation $\epsilon_{(L) \mu}=p_{\mu} / m+v_{\mu}$ and then neglect at high energies those terms containing $v_{\mu}$ factors since $v_{\mu} \simeq O\left(M_{\text {phys }} / E\right)$. Unfortunately, this is not a straightforward procedure due to the gauge structure of the theory which is responsible for cancellations in those amplitudes involving longitudinally polarized gauge bosons, and does not allow us to simply neglect the terms containing $v_{\mu}$ factors.

We can go around this problem using the following relation between amplitudes that we will write symbolically as:

$$
\begin{aligned}
\left(\prod_{i=1}^{n} \epsilon_{(L) \mu_{i}}\right) T\left(\widetilde{W}_{a_{1}}^{\mu_{1}}, \ldots, \widetilde{W}_{a_{n}}^{\mu_{n}} ; A\right) & = \\
& =\sum_{l=0}^{n}(-i)^{l}\left(\prod_{i=1}^{l} v_{\mu_{i}}\right)\left(\prod_{j=l+1}^{n} K_{\alpha_{j}}^{a_{j}}\right) \bar{T}\left(\widetilde{W}_{a_{1}}^{\mu_{1}} \ldots \widetilde{W}_{a_{l}}^{\mu_{l}}, \omega_{\alpha_{l+1}} \ldots \omega_{\alpha_{n}} ; A\right)
\end{aligned}
$$

where we have omitted the irrelevant indices which are supposed to be properly contracted, and we have defined $K_{\alpha}^{a}=\widetilde{L}_{R a}^{(0) \alpha} / M_{p h y s}^{(a)}$. In the right hand side we write $\bar{T}$ since for each $l$ value we carry a sum over all the amplitudes with independent permutations of fields and indices. A very similar relation, but without considering the $K$ factors, was first obtained in [2], the derivation of the formula taking them into account is completely analogous and we do not reproduce it here. These $K$ will modify the final statement of the ET.

When the amplitudes satisfy the unitarity bounds, we can drop at high energies all terms in the RHS of eq.24 but the one with $l=0$ which is precisely that with all external $\widetilde{W}_{L}$ substituted by GB. This step is allowed since the amplitudes will never grow with the energy and therefore those terms which contain $v_{\mu}$ will vanish in the high energy limit. However, when considering effective lagrangians, the amplitudes are obtained perturbatively as a truncated series in the energy so that the same reasoning is no longer valid, and we have to use power counting methods to extract the leading contributions.

In this case we can, in principle, expand the amplitudes as Laurent series in $E / 4 \pi v$ up to a positive power $N$ by fixing the maximum number 
of derivatives in the Lagrangian. However, as we will require these amplitudes to satisfy the Low Energy Theorems (second reference in [14]) in the $M^{2} \ll E^{2}$ regime, we can write the energy negative powers as $(M / E)^{k}$ (To simplify the analysis we have set momentarily $\left.g^{\prime}=0\right)$. Thus we write:

$$
\begin{aligned}
\bar{T}\left(\widetilde{W}_{a_{1}}^{\mu_{1}} \ldots \widetilde{W}_{a_{l}}^{\mu_{l}}, \omega_{\alpha_{l+1}} \ldots \omega_{\alpha_{n}} ; A\right) & \simeq \sum_{k=0}^{N} a_{l}^{k}\left(\frac{E}{4 \pi v}\right)^{k}+\sum_{k=1}^{\infty} a_{l}^{-k}\left(\frac{M}{E}\right)^{k} \\
\left(\prod_{i=1}^{n} \epsilon_{(L) \mu_{i}}\right) T\left(\widetilde{W}_{a_{1}}^{\mu_{1}}, \ldots, \widetilde{W}_{a_{n}}^{\mu_{n}} ; A\right) & \simeq \sum_{k=0}^{N} b^{k}\left(\frac{E}{4 \pi v}\right)^{k}+\sum_{k=1}^{\infty} b^{-k}\left(\frac{M}{E}\right)^{k}
\end{aligned}
$$

These series are formal since the coefficients can contain energy logarithms (for the sake of brevity we have omitted the field indices in $a_{l}^{k}$ and $b^{k}$ ). Furthermore, these coefficients can be expanded perturbatively on $g$, for instance: $a_{l}^{h}=a_{l L}^{h}(1+O(g / 4 \pi))$ where $a_{l L}^{h}$ is the lowest order term in the expansion of $a_{l}^{h}$ in powers of $g$. In most renormalization schemes we have $M \simeq M_{\text {phys }}(1+O(g / 4 \pi))$ which means that we can write as well $K_{\alpha}^{a} \simeq$ $K_{\alpha}^{a(0)}+K_{\alpha}^{a(1)}(g / 4 \pi)+\ldots$. where these coefficients are energy independent. Introducing these expansions in eq.24 and neglecting terms of order $O(M / E)$ and $O(E / 4 \pi v)^{N-n+1}$, we arrive to the following expression:

$$
\begin{aligned}
& \left(\prod_{i=1}^{n} \epsilon_{(L) \mu_{i}}\right) T\left(\widetilde{W}_{a_{1}}^{\mu_{1}}, \ldots, \widetilde{W}_{a_{n}}^{\mu_{n}} ; A\right) \simeq \\
& \simeq\left(\prod_{j=1}^{n} K_{\alpha_{j}}^{a_{j}(0)}\right) \sum_{k=0}^{N-n}\left(a_{0 L}^{k}(1+O(g / 4 \pi))\right)\left(\frac{E}{4 \pi v}\right)^{k}+O\left(\frac{M}{E}\right)+O\left(\frac{E}{4 \pi v}\right)^{N-n+1}
\end{aligned}
$$

which is the statement of the ET for chiral lagrangians (Note that we have omitted for brevity the indices $\alpha$ of $a_{0}$ ). In fact, if we want these approximations to make sense, we have to restrict the values of the energy to the following applicability window:

$$
\begin{array}{r}
M \ll E \ll 4 \pi v=4 \pi M / g \\
g / 4 \pi \ll(E / 4 \pi v)^{N-n+1}
\end{array}
$$

The first two inequalities come from neglecting the $O(M / E)$ and $O(E / 4 \pi v)^{N-n+1}$ terms respectively. The last constraint is needed since we are taking into account the $O(E / 4 \pi v)^{N-n}$ contribution while neglecting that of $O(M / E)$, therefore we expect the former to be much bigger than the latter.

The generalization to the $g^{\prime} \neq 0$ is straightforward due to the fact that $g^{\prime} \ll g$ as well as $M_{Z}^{\text {phys }} \simeq M_{W}^{p h y s} \simeq M^{(a)}$ for any $a$ ( all the different masses 
are of the same order when counting energy powers): we only consider the lowest order of the $a$ coefficients in the $g$ or $g^{\prime}$ expansion so that the same reasoning we had used when $g^{\prime}=0$ is still valid. Thus we can use eq.26 as the precise formulation of the ET for chiral lagrangians.

It is important to remark that this version of the ET states that if we expand in terms of the energy the $S$ matrix element of a process involving $W_{L}$ bosons, and we do the same with the $S$ matrix element obtained by substituting all the longitudinal gauge bosons by their respective GB, we are allowed to approximate the coefficients of the first series not by the corresponding coefficients of the second one, but by their lowest order in terms of $g$ or $g^{\prime}$. Even more, this approximation is expected to be useful when the energy regime satisfies the constraints of eq.27.

\section{Discussion}

In the preceding section we have stated a generalized version of the ET which holds for the chiral lagrangian formalism. As a matter of fact, eq.26 is valid, even when $g^{\prime} \neq 0$, for renormalized amplitudes calculated at any order in the chiral expansion, any choice of the renormalized $\xi^{(a)}$ parameters and any parametrization of the GB coset space. The main difference with the formal version of the ET for regularized amplitudes [11] is contained in the $K$ factors, one per GB, that multiply the RHS of eq.26, that are basically made of the zeroth order of the killing vectors, which selects the GB combination to be eaten by the physical gauge fields, and renormalization constants.

The demonstration has two separated parts: First, from the BRS invariance of the renormalized lagrangian we derive Ward identities to relate renormalized Green functions involving external longitudinal components of gauge bosons to those Green functions where we replace some of this external legs by their corresponding GB as in eqs.14 and 17. The possibility to build a (anti-)BRS invariant renormalized lagrangian although we do not know the precise form of the needed counterterms is due to the absence of anomalies when quantizing the theory. Even though we considered chiral fermions, the usual hypercharge assignments and the fact that $N_{c}=3$ cancel all possible gauge and gauge-gravitational anomalies, as well as the non-perturbative $S U(2)$ discovered by Witten [17]. Some issues related with this point have been recently been discussed in [19]. We have also given a

formalism which is invariant under changes of coordinates in the GB coset, since not only have we built a lagrangian invariant under such changes, but 
the possible reparametrization anomalies are absent since the coset dimension is lower than that of the space-time [18]. Once we have these relations between Green functions we translate them to relations among $S$ matrix elements through the LSZ reduction formula as in eq.22, it is then necessary to rotate the renormalized fields in the lagrangian in order to obtain the physical combinations of fields and set the momenta on shell for the gauge bosons.

Second, the identities thus obtained involve momentum factors that have to be converted in polarization vectors using $\epsilon_{(L) \mu}=p_{\mu} / M+v_{\mu}$ by neglecting at high energies the $v_{\mu}$ factor since it is $O(M / E)$. This step is not straightforward when we are dealing with chiral lagrangians, since the amplitudes are obtained as a chiral expansion in $E / 4 \pi v$ and they can grow with positive powers of the energy violating the unitarity bounds. Therefore we have to perform a power counting analysis to drop the $v_{\mu}$ terms, and obtain the generalized version of the ET. In order to make compatible all the approximations, we have to restrict the possible values of the energy. There will be a high energy bound needed to neglect the $v_{\mu}$ factors as well as a low energy bound in order to make the chiral expansion in energy powers.

The apparent contradiction between these requirements could be avoided thanks to the smallness of the couplings $g / 4 \pi$ and $g^{\prime} / 4 \pi$ which are involved in the expansion at low energies. The actual existence of an applicability window for the theorem given a perturbative order for the calculations can only be tested by detailed computations of different scattering processes. Nevertheless, the validity range of the theorem could be enlarged if one uses non-perturbative techniques to implement unitarity, which would then allow us to avoid the power counting methods, and thus to eliminate the high energy requirements. Among others, these techniques include: dispersion relations and Padé approximants [20], and the large $N$ limit [21], which have been found to work very well when applied to hadron physics.

\section{Conclusion}

In this work we have stated the version of the ET valid for the $\chi \mathrm{PT}$ methods used to describe the GB and gauge boson dynamics of the symmetry breaking sector of the SM. Starting from a gauged NLSM based on the coset $S^{3}=S U(2)_{L} \times S U(2)_{R} / S U(2)_{L+R}$, we have built a renormalized quantum lagrangian which is not only (anti-)BRS invariant but also under reparametrizations of the coset. The existence of these symmetries is due 
to the absence of anomalies in the theory, and they provide us with Ward identities relating renormalized Green functions with external longitudinally polarized gauge bosons and those with GB.

Through a power counting analysis we have been able to formulate the ET version that holds for the renormalized physical amplitudes when calculated by means of a chiral expansion, for any coordinate choice of the GB and for any values of the renormalized $\xi^{(a)}$ parameters. This version states that when expanding in terms of the energy, we can approximate the coefficients of the $S$ matrix element of a process involving $W_{L}$ bosons, not by the corresponding coefficients of the $S$ matrix element obtained by substituting all the longitudinal gauge bosons, but by their lowest order in terms of $g$ or $g^{\prime}$. This fact, and the appearance of the $K$ factors due to the renormalization procedure together with the existence of an upper bound in the energy applicability range, are the main differences with the original formulation of the ET.

In principle, the energy window given in eq.27 for applying simultaneously $\chi \mathrm{PT}$ and the ET at a given loop level can be narrow but the use of non-perturbative techniques could extend it to higher energy regions thus providing a model independent description of the SBS dynamics. Work is in progress in that direction.

\section{Acknowledgements}

This work has been supported in part by the Ministerio de Educación y Ciencia (Spain)(CICYT AEN90-0034). A.D. also thanks the Gregorio del Amo foundation (Universidad Complutense de Madrid) for support and S. Dimopoulos and the Department of Physics of the Stanford University for their kind hospitality during the first part of this work.

\section{Note Added}

When this work was being completed we noticed the appearance of two related preprints on the subject. In [22] the authors arrive, up to the level of the identities obtained from the BRS invariance, to similar results to us for

$g^{\prime}=0$ and a particular parametrization of the GB. However, that work has been critizised in [23] since their analysis does not include the power counting subtleties that arise when the amplitudes are given as truncated series in the energy, as it is customary in the applications of $\chi \mathrm{PT}$. Those considerations 
(see also H.Veltman in [10]) give rise to the upper bound in the applicability range of the ET for chiral lagrangians that has been given in the present work.

\section{References}

[1] J.M. Cornwall, D.N. Levin and G. Tiktopoulos, Phys. Rev. D10 (1974) 1145

C.E.Vayonakis, Lett.Nuovo.Cim.17(1976)383

B.W. Lee, C. Quigg and H. Thacker, Phys. Rev. D16 (1977) 1519

[2] M.S. Chanowitz and M.K. Gaillard, Nucl. Phys. B261(1985) 379

[3] G.J. Gounaris, R. Kogerler and H. Neufeld, Phys. Rev. D34 (1986) 3257

[4] H. Haber and G. Kane, Phys.Rep.117 (1985) 75

[5] E. Farhi and L. Susskind, Phys. Rep. 74 (1981) 277

S. Dimopoulos and L. Susskind, Nucl. Phys. B155 (1979) 237

[6] R.Casalbuoni,S.de Curtis,D.Dominici and R.Gatto, Phys. Lett. B155(1985)95; Nucl. Phys. B282 (1987) 235

[7] S. Weinberg, Physica 96A (1979) 327

J. Gasser and H. Leutwyler, Ann. of Phys. 158 (1984) 142, Nucl. Phys. B250 (1985) 465 and 517

[8] A. Dobado and M.J. Herrero, Phys. Lett. B228 (1989) 495 and B233 (1989) 505

J. Donoghue and C. Ramirez, Phys. Lett. B234 (1990)361

A. Dobado, M.J. Herrero and J. Terrón, Z. Phys. C50 (1991) 205 and Z. Phys. C50 (1991) 465

S. Dawson and G. Valencia, Nucl. Phys. B352 (1991)27

[9] B.Holdom and J. Terning, Phys.Lett. B247 (1990) 88

A. Dobado, D. Espriu and M.J. Herrero Phys.Lett. B255 (1991) 405

M. Golden and L. Randall, Nucl. Phys. B361 (1991) 3 
[10] Y.P.Yao and C.P. Yuan, Phys. Rev. D38 (1988) 2237

J. Bagger and C.Schmidt, Phys. Rev. D41 (1990) 264

H. Veltman, Phys. Rev. D41 (1990) 2294

H.J. He, Y.P. Kuang and X. Li, Phys. Rev. Lett. 69 (1992) 2619

W. B. Kilgore, Phys.Lett. B294 (1992) 257

[11] A. Dobado and J.R. Peláez, Stanford Preprint SU-ITP-93-33, hep-ph 9401202

[12] C. Becchi, A. Rouet and R. Stora, Comm. Math. Phys. 42(1975) 127

[13] L. Baulieu and J.Thierry-Mieg Nucl. Phys. B197 (1982) 477

L. Alvarez-Gaumé and L. Baulieu, Nucl. Phys. B212 (1985) 255

L. Baulieu, Phys. Rep. 129 (1985) 1

[14] P. Sikivie et al., Nucl. Phys. B173 (1980) 189

M. S. Chanowitz, M. Golden and H. Georgi Phys.Rev. D36 (1987) 1490

[15] J. Charap, Phys. Rev. D2 (1970)1115

I.S. Gerstein, R. Jackiw, B. W. Lee and S. Weinberg, Phys. Rev. D3 (2486)1971

J. Honerkamp, Nucl. Phys. B36 (1972)130

Quantum Field Theory and Critical Phenomena, J. Zinn-Justin, Oxford University Press, New York, (1989)

L. Tararu, Phys. Rev. D12 (1975)3351

D. Espriu and J. Matias, Preprint UB-ECM-PF 93/15

[16] T. Appelquist and C. Bernard, Phys. Rev. D22 (1980) 200

A. C. Longhitano, Nucl.Phys. B188 (1981) 118

[17] E. Witten,Phys. Lett. B117 (1982)324

[18] L. Alvarez-Gaumé and P. Ginsparg, Nucl.Phys. B262(1985) 439

[19] J.F.Donoghue,Phys. Lett. B301 (1993)372

P.B.Pal,Phys. Lett. B321 (1994)229

W.B.Kilgore, Phys. Lett. B323 (1994)161

[20] Tran N. Truong, Phys. Rev. D61 (1988)2526

A. Dobado, M.J. Herrero and J.N. Truong, Phys. Lett. B235 
(1990) 134

T.N.Truong, Phys. Rev. Lett. 67 (1991)2260

A. Dobado and J.R. Peláez, Phys. Rev. D47(1992)4883

[21] C.J.C. Im, Phys. Lett. B281 (1992)357

A. Dobado and J.R. Peláez, Phys. Lett. B286 (1992)136

M. J. Dugan and M. Golden, Phys.Rev. D48 (1993)4375

[22] H.J.He, Y.P.Kuang, and X.Li, Tsinghua preprint TUIMP-TH94/56, hep-ph/9403283

[23] C.Grosse-Knetter, I.Kuss. Bielefield preprint BI-TP 94/10, hep$\mathrm{ph} / 9403291$ 